TRANSACTIONS OF THE

AMERICAN MATHEMATICAL SOCIETY

Volume 356, Number 7, Pages 2649-2661

S 0002-9947(03)03250-

Article electronically published on October 29, 2003

\title{
SUBVARIETIES OF GENERAL TYPE ON A GENERAL PROJECTIVE HYPERSURFACE
}

\author{
GIANLUCA PACIENZA
}

\begin{abstract}
We study subvarieties of a general projective degree $d$ hypersurface $X_{d} \subset \mathbf{P}^{n}$. Our main theorem, which improves previous results of L. Ein and C. Voisin, implies in particular the following sharp corollary: any subvariety of a general hypersurface $X_{d} \subset \mathbf{P}^{n}$, for $n \geq 6$ and $d \geq 2 n-2$, is of general type.
\end{abstract}

\section{INTRODUCTION}

Let $X_{d} \subset \mathbf{P}^{n}$ be a general (in the countable Zariski topology) complex projective hypersurface of degree $d$. The study of the geometry of $k$-dimensional subvarieties of $X_{d}$ in terms of $k, n$ and $d$ has received much attention in the last 15 years (see [C1], [E1] and [E2], [X1] and [X2], [V1] and [V2], [CL], [CLR], $\mathrm{P}$, [C2], CR ). In particular, this study is related to the hyperbolicity of the hypersurface $X_{d} \subset \mathbf{P}^{n}$. Recall that a compact complex manifold $M$ is said to be hyperbolic (in the sense of Brody or Kobayashi) if there are no nonconstant entire holomorphic maps $f: \mathbb{C} \rightarrow M$. S. Lang conjectured (cf. $[\mathrm{L}$, Conjecture 5.6) that, in the case of a projective variety $V$, the notion of hyperbolicity has an algebraic characterization, namely $V$ is hyperbolic if and only if any subvariety $Y$ of $V$ is of general type (that is, if $Y$ is smooth, some multiple of the canonical bundle of $Y$ gives a projective embedding of a non-empty Zariski open subset of $Y$. If $Y$ is singular, then it is said to be of general type if some desingularization of $Y$ has this property). Notice that if any subvariety $Y$ of $V$ is of general type, then in particular $V$ does not contain rational curves or abelian subvarieties - a condition which is of course implied by the hyperbolicity. In this paper we focus our attention on the case of a general projective hypersurface $X_{d} \subset \mathbf{P}^{n}$, and give a sharp bound on its degree $d$ for it to satisfy the algebraic property above that, conjecturally, should be equivalent to the hyperbolicity of $X_{d}$.

This problem has been studied by L. Ein in [E1] and [E2], where, generalizing a previous result by H. Clemens [C1], he proves in particular that whenever $d \geq 2 n-k$, for $n \geq 3$, then any $k$-dimensional subvariety $Y$ of the general $X_{d} \subset \mathbf{P}^{n}$ has nonzero geometric genus, and if the inequality is strict then $Y$ is of general type. Ein's result, which concerns more generally subvarieties of general complete intersections in an arbitrary smooth projective variety, has been improved by one, in the case of projective hypersurfaces, by C. Voisin ( $\mathrm{V1}$, [V2]), who proves the following theorem.

Received by the editors August 1, 2002 and, in revised form, October 11, 2002.

2000 Mathematics Subject Classification. Primary 14J70, 14K12, 14C99; Secondary 32Q45. 
Theorem (Voisin). Let $X_{d} \subset \mathbf{P}^{n}$ be a general hypersurface of degree $d \geq 2 n-k-1$, where $k$ is an integer such that $1 \leq k \leq n-3$. Then any $k$-dimensional subvariety $Y$ of $X$ has nonzero geometric genus, and if the inequality is strict then $Y$ is of general type.

Our main result is the following:

Theorem 1.1. Let $X_{d} \subset \mathbf{P}^{n}$ be a general complex projective hypersurface of degree d. Let $Y \subset X_{d}$ be a $k$-dimensional subvariety with a desingularization $\tilde{Y} \stackrel{j}{\rightarrow} Y$ such that $h^{0}\left(\tilde{Y}, K_{\tilde{Y}} \otimes j^{*} \mathcal{O}_{\mathbf{P}^{n}}(-1)\right)=0$. If the inequalities

$$
d-1 \geq \max \left\{\frac{7 n-3 k-3}{4}, \frac{3 n-k+1}{2}\right\}
$$

and

$$
\frac{d(d-3)}{2} \geq 2 n-k-3
$$

are satisfied, then $Y$ is contained in the locus covered by the lines of $X$.

Since the dimension of the locus spanned by the lines on a general hypersurface $X_{d}$ is equal to $(2 n-2)-d$, it follows from Theorem 1.1 that for any subvariety $Y$ of $X_{d} \subset \mathbf{P}^{n}$, whenever $d \geq 2 n-2$ and $n \geq 6$, the canonical bundle of a desingularization $j: \tilde{Y} \rightarrow Y$ is the sum of the effective divisor $K_{\tilde{Y}} \otimes j^{*} \mathcal{O}_{\mathbf{P}^{n}}(-1)$ and of $j^{*} \mathcal{O}_{\mathbf{P}^{n}}(1)$, which is very ample on an open subset, so we obtain

Corollary 1.2. Any subvariety of a general hypersurface $X_{d} \subset \mathbf{P}^{n}$, with $d \geq 2 n-2$ and $n \geq 6$, is of general type.

Corollary 1.2 is sharp, since general hypersurfaces of degree $2 n-3$ contain a finite number of lines (and, by $[\mathrm{P}]$, lines are the only rational curves allowed on the general $X_{2 n-3} \subset \mathbf{P}^{n}$, for $n \geq 6$ ).

The weird looking numerical hypotheses (1) and (2) of Theorem 1.1 are needed in order to control the positivity of the twisted exterior powers of the bundle $M_{\mathbf{P}^{n}}^{d}$ (resp. $M_{G}^{d}$ ) over $\mathbf{P}^{n}$ (resp. over the Grassmannian of lines in $\mathbf{P}^{n}$ ), which are defined in $\S 2$. This control will appear to be a crucial point in the proof (cf. Lemmas 3.1 4.1 and 4.3). The proof of Theorem 1.1 makes use of the powerful variational approach introduced by C. Voisin in [V1] and [V2], and adopted by the author in [P] to study the geometry of subvarieties having geometric genus zero on a general hypersurface. These methods have been strengthtened more recently by $\mathrm{H}$. Clemens and Z. Ran (see [C2] and [CR]) to study in greater generality subvarieties $Y$ of $X$ with desingularizations $j: \tilde{Y} \rightarrow Y$ satisfying $h^{0}\left(\tilde{Y}, K_{\tilde{Y}} \otimes j^{*} \mathcal{O}_{\mathbf{P}^{n}}(a)\right)=0$, for some integer $a \geq 0$.

The proof is naturally divided into two parts. First, following an idea that goes back to Voisin $[\mathrm{V2}$, under a technical numerical hypothesis, it is possible to see that through each point of $Y$ there is a line which intersects $X$ set-theoretically in at most two points. Precisely, we prove

Proposition 1.3. Let $X_{d} \subset \mathbf{P}^{n}$ be a general hypersurface whose degree d satisfies the numerical condition (11) of Theorem 1.1, and $Y \subset X_{d}$ a subvariety of dimension $k$ such that $h^{0}\left(\tilde{Y}, K_{\tilde{Y}} \otimes j^{*} \mathcal{O}_{\mathbf{P}^{n}}(-1)\right)=0$, where $j: \tilde{Y} \rightarrow Y$ is a desingularization. Then, for some $r \geq 1, Y$ is contained in the sublocus $\Delta_{(r, d-r), X}$ of $X_{d}$ defined as

$$
\Delta_{(r, d-r), X}:=\left\{x \in X_{d}: \exists \text { a line } \ell \text { s.t. } \ell \cap X_{d}=r \cdot x+(d-r) \cdot x^{\prime}, x^{\prime} \in X_{d}\right\} .
$$


(This result, under a different numerical hypothesis, can also be found in [CR]).

The second part of the proof of Theorem 1.1 deals with the study of the locus $\Delta_{(r, d-r), X}$. For this, we use two explicit desingularizations of $\Delta_{(r, d-r), X}$, whose canonical bundles are easily computable. Then, in both cases, the key point is the construction of a globally generated subbundle contained in the exterior powers of the (twisted) tangent bundle of the family of the desingularizations. This fact will allow us to obtain the following proposition, which concludes the proof of Theorem 1.1:

Proposition 1.4. Let $X_{d} \subset \mathbf{P}^{n}$ be a general hypersurface of degree d satisfying the numerical condition (21) of Theorem 1.1. Let $Y \subset \Delta_{(r, d-r), X}$ be a subvariety of dimension $k$, and $j: \tilde{Y} \rightarrow Y$ a desingularization such that $h^{0}\left(\tilde{Y}, K_{\tilde{Y}} \otimes j^{*} \mathcal{O}_{\mathbf{P}^{n}}(-1)\right)=$ 0 . Then $Y$ is contained in the locus of lines of $X_{d}$.

\section{Preliminaries}

We will follow the notation already used in $[\mathrm{P}]$, which we recall below.

Notation. $S^{d}:=H^{0}\left(\mathbf{P}^{n}, \mathcal{O}_{\mathbf{P}^{n}}(d)\right)$;

$S_{x}^{d}:=H^{0}\left(\mathbf{P}^{n}, \mathcal{I}_{x} \otimes \mathcal{O}_{\mathbf{P}^{n}}(d)\right)$;

$N:=h^{0}\left(\mathbf{P}^{n}, \mathcal{O}_{\mathbf{P}^{n}}(d)\right)=\operatorname{dim} S^{d}$;

$\mathcal{X} \subset \mathbf{P}^{n} \times S^{d}$ will denote the universal hypersurface of degree $d$;

$X_{F} \subset \mathbf{P}^{n}$ is the fiber of the family $\mathcal{X}$ over $F \in S^{d}$, i.e. the hypersurface defined by $F$.

Let $U \rightarrow S^{d}$ be an étale map and $\mathcal{Y} \subset \mathcal{X}_{U}$ a reduced and irreducible subscheme of relative dimension $k$ (in the following, by abuse of notation, we will often omit the étale base change). Let $\tilde{\mathcal{Y}} \rightarrow \mathcal{Y}$ be a desingularization and $\tilde{\mathcal{Y}} \stackrel{j}{\rightarrow} \mathcal{X}_{U}$ the natural induced map. We may obviously assume $\mathcal{Y}$ invariant under some lift of the action of $G L(n+1)$ (recall that $g \in G L(n+1)$ acts on the product $\mathbf{P}^{n} \times S^{d}$ as follows: $\left.g(x, F)=\left(g(x),\left(g^{-1}\right)^{*} F\right)\right)$. Let $\pi: \mathcal{X} \rightarrow \mathbf{P}^{n}$ be the projection on the first component and $T_{\mathcal{X}}^{\text {vert }}\left(\right.$ resp. $\left.T_{\mathcal{Y}}^{\text {vert }}\right)$ the vertical part of $T_{\mathcal{X}}\left(\right.$ resp. $\left.T_{\mathcal{Y}}\right)$ w.r.t. $\pi$, i.e. $T_{\mathcal{X}}^{\text {vert }}\left(\right.$ resp. $\left.T_{\mathcal{Y}}^{\text {vert }}\right)$ is the sheaf defined by

$$
\begin{gathered}
0 \rightarrow T_{\mathcal{X}}^{\text {vert }} \rightarrow T \mathcal{X} \stackrel{\pi_{*}}{\rightarrow} T \mathbf{P}^{n} \rightarrow 0 \\
\left(\text { resp. } \quad 0 \rightarrow T_{\mathcal{Y}}^{\text {vert }} \rightarrow T \mathcal{Y} \stackrel{\pi_{*}}{\longrightarrow} T \mathbf{P}^{n}\right) .
\end{gathered}
$$

Remark 2.0.1. Let $\mathcal{Y}$ be a subscheme of $\mathcal{X} \subset \mathbf{P}^{n} \times S^{d}$ of relative dimension $k$ and invariant under the action of $G L(n+1)$. Then:

(i) the map $T \mathcal{Y} \stackrel{\pi_{*}}{\longrightarrow} T \mathbf{P}^{n}$ is surjective, and hence

$$
\operatorname{codim}_{T_{\mathcal{X},(y, F)}^{v e r t}} T_{\mathcal{Y},(y, F)}^{v e r t}=\operatorname{codim}_{\mathcal{X}} \mathcal{Y}=n-k-1 ;
$$

(ii) $T_{\mathcal{Y},(y, F)}^{v e r t}$ contains the vertical part of the tangent space to the orbit of the point $(y, F)$ under the action of $G L(n+1)$, i.e.

$$
T_{\mathcal{Y},(y, F)}^{v e r t} \supset\left\langle S_{y}^{1} \cdot J_{F}^{d-1}, F\right\rangle,
$$

where $J_{F}^{d-1}$ is the Jacobian ideal of $F$.

Let $d$ be a positive integer. Consider the bundle $M_{\mathbf{P}^{n}}^{d}$ defined by the exact sequence

$$
0 \rightarrow M_{\mathbf{P}^{n}}^{d} \rightarrow S^{d} \otimes \mathcal{O}_{\mathbf{P}^{n}} \stackrel{e v}{\longrightarrow} \mathcal{O}_{\mathbf{P}^{n}}(d) \rightarrow 0
$$


whose fiber at a point $x$ identifies by definition to $S_{x}^{d}$. From the inclusion $\mathcal{X} \hookrightarrow$ $\mathbf{P}^{n} \times S^{d}$ we get the exact sequence

$$
0 \rightarrow T \mathcal{X}_{X_{F}} \rightarrow T \mathbf{P}^{n}{ }_{\mid X_{F}} \oplus\left(S^{d} \otimes \mathcal{O}_{X_{F}}\right) \rightarrow \mathcal{O}_{X_{F}}(d) \rightarrow 0,
$$

which combined with (3) gives us

$$
0 \rightarrow M_{\mathbf{P}^{n} \mid X_{F}}^{d} \rightarrow T \mathcal{X}_{\mid X_{F}} \rightarrow T \mathbf{P}^{n}{ }_{\mid X_{F}} \rightarrow 0 .
$$

In other words, $M_{\mathbf{P}^{n} \mid X_{F}}^{d}$ identifies to the vertical part of $T_{\mathcal{X}} \otimes \mathcal{O}_{X_{F}}$ with respect to the projection to $\mathbf{P}^{n}$.

Let $G:=\operatorname{Grass}(1, n)$ be the Grassmannian of lines in $\mathbf{P}^{n}, \mathcal{O}_{G}(1)$ the line bundle on $G$ giving its Plücker polarization, and $\mathcal{E}_{d}$ the $d^{\text {th }}$ symmetric power of the dual of the tautological subbundle on $G$. Recall that the fibre of $\mathcal{E}_{d}$ at a point $[\ell]$ is, by definition, given by $H^{0}\left(\ell, \mathcal{O}_{\ell}(d)\right)$.

Let $M_{G}^{d}$ be the vector bundle on $G$ defined as the kernel of the evaluation map:

$$
0 \rightarrow M_{G}^{d} \rightarrow S^{d} \otimes \mathcal{O}_{G} \rightarrow \mathcal{E}_{d} \rightarrow 0 .
$$

Notice that the fiber of $M_{G}^{d}$ at a point [ $\left.\ell\right]$ is equal to $H^{0}\left(\mathcal{I}_{\ell}(d)\right)$.

The bundles $M_{\mathbf{P}^{n}}^{d}$ and $M_{G}^{d}$ satisfy the following positivity properties, which will often be used in what follows:

Lemma 2.1. (i) $M_{\mathbf{P}^{n}}^{d} \otimes \mathcal{O}_{\mathbf{P}^{n}}(1)$ is generated by its global sections.

(ii) $M_{G}^{d} \otimes \mathcal{O}_{G}(1)$ is generated be its global sections.

Proof. See, for instance, [P].

\section{A FIRST REDUCTION}

Let $Y_{F} \subset X_{F}$ be a general ( $k$-dimensional) fiber of the subfamily $\mathcal{Y} \subset \mathcal{X}_{U}$, and $\tilde{Y}_{F} \stackrel{j}{\rightarrow} Y_{F}$ its desingularization. By abuse of notation, we will often write $K_{\tilde{Y}_{F}}(-1)$ instead of $\left.K_{\tilde{Y}_{F}} \otimes j^{*} \mathcal{O}_{\mathbf{P}^{n}}(-1)\right)$.

Recall now the following isomorphisms:

(i) $\Omega_{\tilde{\mathcal{Y}}_{\mid \tilde{Y}_{F}}}^{N+k} \cong K_{\tilde{Y}_{F}}$;

(ii) $\left(\bigwedge^{n-1-k} T \mathcal{X}_{U \mid X_{F}}\right) \otimes K_{X_{F}} \cong \Omega_{\mathcal{X}_{U} \mid X_{F}}^{N+k}$.

Set $c:=n-1-k=\operatorname{codim}_{X_{F}} Y_{F}$. Using (i) and (ii), from the natural morphism $\Omega_{\mathcal{X}_{U}}^{1} \rightarrow \Omega_{\tilde{\mathcal{Y}}}^{1}$, we get a map

$$
\left(\bigwedge^{c} T \mathcal{X}_{U \mid X_{F}}\right) \otimes K_{X_{F}} \cong \Omega_{\mathcal{X}_{U}{ }_{\mid X_{F}}+k} \rightarrow \Omega_{\tilde{\mathcal{Y}}}^{N+k}{ }_{\mid \tilde{Y}_{F}} \cong K_{\tilde{Y}_{F}},
$$

and hence, after tensoring by $\mathcal{O}_{X_{F}}(-1)$,

$$
\left(\bigwedge^{c} T \mathcal{X}_{U \mid X_{F}}\right) \otimes K_{X_{F}}(-1) \longrightarrow K_{\tilde{Y}_{F}}(-1) .
$$

Now taking global sections in (6) and using (4) we have the following commutative diagram

$$
\begin{gathered}
H^{0}\left(\bigwedge^{c} T \mathcal{X}_{U \mid X_{F}} \otimes K_{X_{F}}(-1)\right) \\
\cup
\end{gathered}
$$


By hypothesis, we have that the composite map in (7) is identically zero. This, by the $G L(n+1)$-invariance of $\mathcal{Y}$, implies that $T_{\mathcal{Y},(y, F)}^{v e r t}$ is then contained in the base locus of $H^{0}\left(\bigwedge^{c} M_{\mathbf{P}^{n} \mid X_{F}}^{d} \otimes K_{X_{F}}(-1)\right)$, considered as the space of sections of a line bundle on the Grassmannian of codimension $c$ subspaces of $T^{\text {vert }} \mathcal{X}_{\mid X_{F}}$.

The generalization presented in $\mathrm{C} 2$ of the variational approach introduced by C. Voisin in [V1] and [V2], and applied by the author in [P] starts with a sharp algebraic study of the base locus of the bundles $\bigwedge^{c} M_{\mathbf{P}^{n}}^{d}(b)$. Precisely, we will use the following:

Lemma 3.1 (C2). Suppose d satisfies the numerical condition (11) of Theorem 1.1. Let $A$ be a codimension c subspace of $S_{x}^{d}=\left(T_{\mathcal{X},(x, F)}\right)^{\text {vert }}$ which is in the base locus of $H^{0}\left(\bigwedge^{c} M_{\mathbf{P}^{n}}^{d}(d-n-2)\right)$. Then there exists a line $\ell_{A}$ passing through $x$ such that

$$
r k \frac{A}{A \cap H^{0}\left(\mathcal{I}_{\ell_{A}}(d)\right)} \leq n+1
$$

Proof. In [C2 the base locus of $H^{0}\left(\bigwedge^{c} M_{\mathbf{P}^{n}}^{d}(d-n-1+a)\right)$ is studied for $a \geq 0$ (this is the point where Lemma 2.1, (i) is used). Here we simply remark that the arguments presented in [C2] also apply to the case $a=-1$. For the reader's convenience we outline the idea of the proof (for the details, see [C2]). The main point in $[\mathrm{V2}$ and $[\mathrm{P}]$ was to produce, by Koszul complex techniques, explicit global sections of the bundle $\bigwedge^{c} M_{\mathbf{P}^{n}}^{d}(b)$, for the integers $b, c, d$ considered there. This was used to deduce that, for a generic polynomial $P \in S^{d-1}$, the multiplication map

$$
\begin{aligned}
m_{P, A}: \quad S_{x}^{1} & \rightarrow S_{x}^{d} / A, \\
L & \mapsto L \cdot P \bmod A,
\end{aligned}
$$

has rank one. H. Clemens considers more generally in [C2] the smallest integer $s \geq 0$ such that rank of the multiplication map

$$
\begin{aligned}
m_{P, A, s}: S_{x}^{1} & \rightarrow S_{x}^{d} /\left(A+Q_{1} \cdot S_{x}^{1}+\ldots+Q_{s} \cdot S_{x}^{1}\right), \\
L & \mapsto L \cdot P \bmod \left(A+Q_{1} \cdot S_{x}^{1}+\ldots+Q_{s} \cdot S_{x}^{1}\right), \\
& P, Q_{1}, \ldots, Q_{s} \text { are generic polynomials in } S^{d-1},
\end{aligned}
$$

is one. Then, as in [V2] and $[\mathrm{P}$, an infinitesimal argument applies. Namely, recall that if $V$ and $W$ are vector spaces, and $Z_{k}:=\{\phi \in \operatorname{Hom}(V, W): \operatorname{rank} \phi \leq k\}$, then

$$
T_{Z_{k}, \phi}=\{\psi \in \operatorname{Hom}(V, W): \psi(\operatorname{ker} \phi) \subset \operatorname{Im} \phi\} .
$$

Applying this to the map $m_{P, A, s}$, we obtain that, for any $R \in S^{d-1}$,

$$
R \cdot \operatorname{Ker} m_{P, A, s} \bmod \left(A+Q_{1} \cdot S_{x}^{1}+\ldots+Q_{s} \cdot S_{x}^{1}\right) \subset \operatorname{Im} m_{P, A, s}
$$

i.e.

$$
H^{0}\left(\mathcal{I}_{\ell_{A}}(d)\right) \subset A+Q_{1} \cdot S_{x}^{1}+\ldots+Q_{s} \cdot S_{x}^{1}+P \cdot S_{x}^{1}
$$

where $\ell_{A}$ is the line determined by $\operatorname{Ker} m_{P, A, s}$. To complete the proof it remains to verify that the line is independent of the choice of the polynomials, and that, under the hypothesis (1), the integer $s$ is such that $A$ and $H^{0}\left(\mathcal{I}_{\ell_{A}}(d)\right)$ satisfy (8).

Since the map (71) vanishes, Lemma [3.1] applies to the tangent space $T_{\mathcal{Y},(y, F)}^{v e r t}$. Then, at a generic point $(y, F) \in \mathcal{Y}$, the tangent space $T_{\mathcal{Y},(y, F)}^{v e r t}$ contains a subspace 
$T \subset H^{0}\left(\mathcal{I}_{\ell_{(y, F)}}(d)\right)$, where $\ell_{(y, F)}$ is a line through $y$ and $T$ satisfies

$$
r k \frac{T_{\mathcal{Y},(y, F)}^{v e r t}}{T} \leq n+1
$$

We now verify an easy fact:

Lemma 3.2. The tangent space $T_{\mathcal{Y},(y, F)}^{v e r t}$ cannot contain two subspaces $T$ and $T^{\prime}$ of ideals of different lines $\ell \neq \ell^{\prime}$ and satisfying (10).

Proof. Indeed, if this were the case, by the surjectivity of

$$
H^{0}\left(\mathcal{I}_{\ell}(d)\right) \oplus H^{0}\left(\mathcal{I}_{\ell^{\prime}}(d)\right) \rightarrow S_{y}^{d}
$$

and the numerical condition (10), then $T_{\mathcal{Y},(y, F)}^{v e r t}$ would contain a subspace of $S_{y}^{d}$ of codimension at most $2(n+c+1-d)$. Now, by Remark 2.0.1, (i), we have

$$
c=\operatorname{codim}_{T_{\mathcal{X},(y, F)}^{v e r t}} T_{\mathcal{Y},(y, F)}^{v e r t}=\operatorname{codim}_{\mathcal{X}} \mathcal{Y} \leq 2(n+c+1-d),
$$

which is equivalent to

$$
d \leq \frac{3 n-k}{2},
$$

and the last inequality is impossible because of (1).

Then, we can consider the distribution $\mathcal{T} \subset T_{\mathcal{Y}}^{\text {vert }}$, pointwise given by the $T$ 's. This distribution turns out to have the following properties.

Proposition 3.3. The distribution $\mathcal{T} \subset T_{\mathcal{Y}}^{\text {vert }}$ is integrable, and the natural map $\phi: \mathcal{Y} \rightarrow G(1, n)$, associating to $(y, F)$ the line determined by $T \subset H^{0}\left(\mathcal{I}_{\ell_{(y, F)}}(d)\right)$, is constant along the leaves of the corresponding foliation.

Proof. The proof goes along the lines of [V2], Lemmas 3 and 4, and of $[\mathrm{P}]$, Lemma 3.3. For the detailed proof in the general case, see [C2]. Again, for the reader's convenience, we sketch it below. Consider the bracket map

$$
\Psi: \bigwedge^{2} \mathcal{T} \rightarrow T_{\mathcal{Y}}^{\text {vert }} / \mathcal{T} \subset T_{\mathcal{X}}^{\text {vert }}{ }_{\mid \mathcal{Y}} / \mathcal{T}
$$

which is given at the point $(y, F)$ by

$$
\psi: \bigwedge^{2} T_{\ell_{(y, F)}} \rightarrow T_{\mathcal{Y},(y, F)}^{v e r t} / T_{\ell_{(y, F)}} \hookrightarrow H^{0}\left(\mathcal{O}_{\ell_{(y, F)}}(d)(-y)\right) .
$$

Now, choose coordinates on $\mathbf{P}^{n}$ such that $\ell:=\ell_{(y, F)}=\left\{X_{2}=\ldots=X_{n}=0\right\}$ and $y=[1,0, \ldots, 0]$. Note that, since $y \in \ell, \phi_{*}\left(T_{\mathcal{Y},(y, F)}^{v e r t}\right)$ is contained in $H^{0}\left(N_{\ell / \mathbf{P}^{n}}(-y)\right)$ $\left(\phi_{*}\right.$ is the differential of $\phi$ at $\left.(y, F)\right)$. One verifies that

$$
\psi(A \wedge B)=A \cdot \phi_{*}(B)-B \cdot \phi_{*}(A), A, B \in T_{\ell_{(y, F)}},
$$

where the bilinear map $(a, b) \mapsto a \cdot b$ is explicitly given by

$$
P \cdot\left(X_{1} \sum_{i=2}^{n} b_{i} \frac{\partial}{\partial X_{i}}\right)=\sum_{i=2}^{n} b_{i} X_{1}\left(\frac{\partial P}{\partial X_{i}}\right)_{\mid \ell} \in H^{0}\left(\mathcal{O}_{\ell}(d)(-y)\right) .
$$

A key linear algebra lemma allows to prove that $\phi_{*}$ is zero, so the proposition follows from (11) and from the Frobenius theorem.

Using this, we will prove, via the $G L(n+1)$-invariance of $\mathcal{Y}$, that $F_{\mid \ell_{(y, F)}}$ has, set-theoretically, at most two zeroes. 
Proof of Proposition 1.3. Let $(y, F)$ be a general point of $\mathcal{Y}$. Let $\ell$ be the line through $y$, and $T$ the subspace of $H^{0}\left(\mathcal{I}_{\ell}(d)\right)$ contained in $T_{\mathcal{Y},(y, F)}^{\text {vert }}$ and satisfying (10). Consider the following diagram:

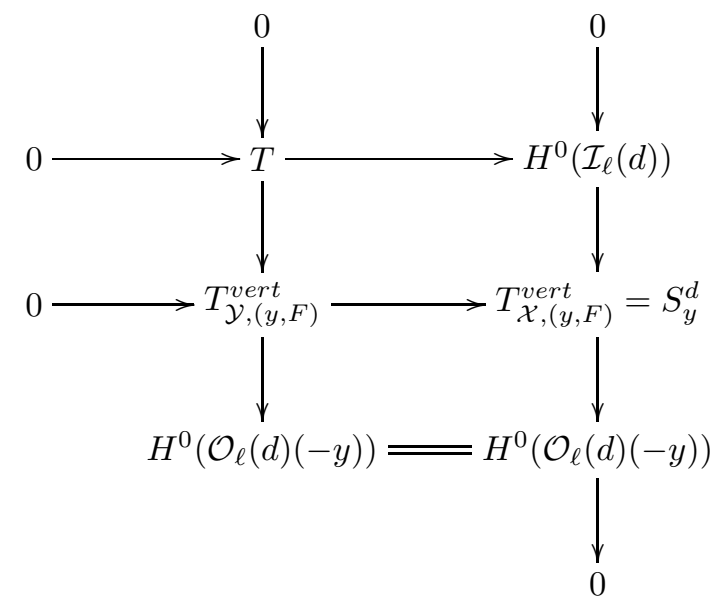

By (10) we have

$$
\operatorname{dim} \operatorname{Im}\left(T_{\mathcal{Y},(y, F)}^{\text {vert }} \rightarrow H^{0}\left(\mathcal{O}_{\ell}(d)(-y)\right)\right) \leq n+1
$$

On the other hand, by Remark 2.0.1, (ii), the vertical tangent space $T_{\mathcal{Y},(y, F)}^{v e r t}$ contains $S_{y}^{1} \cdot J_{F}^{d-1}$ and $F$ itself. Take coordinates $X_{0}, \ldots, X_{n}$ on $\mathbf{P}^{n}$ such that $y=[1,0, \ldots, 0]$ and $\ell:=\ell_{(y, F)}=\left\{X_{2}=\ldots=X_{n}=0\right\}$. Since $\phi$ is constant along the leaves of the foliation, we can generically choose a polynomial $G$ in the leaf integrating $T$ so that the $(n-1)$ elements $X_{1} \frac{\partial G}{\partial X_{i}}, i \geq 2$, are independent modulo the subpace

$$
K:=\left\langle G_{\mid \ell}, X_{1}\left(\frac{\partial G}{\partial X_{0}}\right)_{\mid \ell}, X_{1}\left(\frac{\partial G}{\partial X_{1}}\right)_{\mid \ell}\right\rangle \subset H^{0}\left(\ell, \mathcal{O}_{\ell}(d) \otimes \mathcal{I}_{y}\right),
$$

which is uniquely determined by $F_{\mid \ell}$ and hence is constant along the leaf integrating $T$. By (13), this implies $\operatorname{dim} K \leq 2$; that is,

$$
F_{\mid \ell}=\alpha X_{1}^{r} L^{d-r}
$$

for some $r \geq 1$ and some linear form $L$ on $\ell$.

We are then led to study the locus $\Delta_{(r, d-r), F}$. This will be done in the last section.

\section{The Bicontact locus $\Delta_{(r, d-r), F}$}

Let $X_{F} \subset \mathbf{P}^{n}$ be a general hypersurface of degree $d$ satisfying (11), and $Y_{F} \subset X_{F}$ a $k$-dimensional subvariety whose desingularization $\tilde{Y}$ is such that $h^{0}\left(\tilde{Y}, K_{\tilde{Y}}(-1)\right)=$ 0 . Then, by Proposition 1.3. we know that $Y_{F}$ is contained in $\Delta_{(r, d-r), X_{F}} \subset X_{F}$, the $(2 n-d)$-dimensional subvariety of points $x$ of $X_{F}$ through which there is an osculating line $\ell$ intersecting $X_{F}$ at most at one other point, i.e. $\ell \cap X_{F}=r \cdot x+$ $(d-r) \cdot x^{\prime}, x^{\prime} \in X_{F}$. In what follows we will write $\Delta_{(r, d-r), F}$ instead of $\Delta_{(r, d-r), X_{F}}$. To prove our theorem, we study two explicit desingularizations of $\Delta_{(r, d-r), F}$, which have been used in $\mathrm{V2}$, both given in terms of the zero locus of a section of a vector bundle. Thus we compute, by adjunction, the canonical bundle of such a 
desingularization. Then, again, we adopt a variational approach and construct, in both cases, a subbundle contained in the exterior powers of the (twisted) tangent bundle to the family of the desingularizations. A positivity result, namely the global generation of this subbundle, allows us to conclude the proof.

Case 1: $r \geq 2$ and $d-r \geq 2$.

Let $G:=\operatorname{Gr}(1, n)$ be the Grassmannian of lines in $\mathbf{P}^{n}$. Let $\mathcal{O}_{G}(1)$ be the line bundle on $G$ which gives the Plücker embedding. Let $Z$ be the blow-up along the diagonal $\Delta$ of the product $\mathbf{P}^{n} \times \mathbf{P}^{n}$ with projections

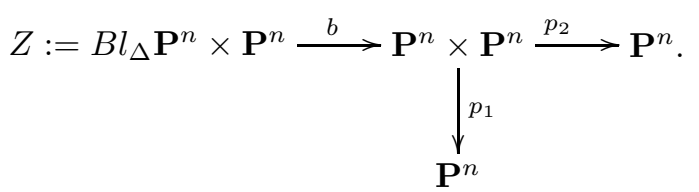

Consider the map

$$
\begin{aligned}
f: Z & \rightarrow G r(1, n), \\
z & \mapsto \ell_{z},
\end{aligned}
$$

where $\ell_{z}$ is the line determined by $z$. Let $\tilde{p}_{i}:=p_{i} \circ b$, for $i=1,2$, and consider the line bundles on $Z$ defined as follows: $H_{i}:=\tilde{p}_{i}{ }^{*} \mathcal{O}_{\mathbf{P}^{n}}(1)$ and $L:=f^{*} \mathcal{O}_{G}(1)$. The variety $Z$ comes together with a projective bundle: $\mathbf{P} \stackrel{\pi}{\longrightarrow} Z$, and we define $\mathcal{E}_{d}:=\pi_{*} \mathcal{O}_{\mathbf{P}}(d)$. Notice that the fibre of $\mathcal{E}_{d}$ at $z$ is equal to $H^{0}\left(\ell_{z}, \mathcal{O}_{\ell_{z}}(d)\right)$. Consider the line bundle $\mathcal{L}_{r, d-r} \subset \mathcal{E}_{d}$, whose fibre at $z \in Z$ is given by the one-dimensional space of polynomials $P \in H^{0}\left(\ell_{z}, \mathcal{O}_{\ell_{z}}(d)\right)$ vanishing at $x$ to the order $r$ and at $y$ to the order $d-r$, where $(x, y)=b(z) \in \mathbf{P}^{n} \times \mathbf{P}^{n}$. Define $\mathcal{F}_{r, d-r}:=\mathcal{E}_{d} / \mathcal{L}_{r, d-r}$. To any polynomial $F \in S^{d}$ we can associate a section $\sigma_{F} \in H^{0}\left(Z, \mathcal{E}_{d}\right)$ whose value at a point $z$ is exactly the polynomial $F_{\mid \ell_{z}} \in \mathcal{E}_{d \mid z}$, and we will denote by $\bar{\sigma}_{F}$ its image in $H^{0}\left(Z, \mathcal{F}_{r, d-r}\right)$. Then we define $\tilde{\Delta}_{(r, d-r), F}:=V\left(\bar{\sigma}_{F}\right)$. By construction, we have $\tilde{p}_{1}\left(\tilde{\Delta}_{(r, d-r), F}\right)=\Delta_{(r, d-r), F}$. Since $\mathcal{F}_{r, d-r}$ is generated by its global sections, the variety $\tilde{\Delta}_{(r, d-r), F}$ is smooth and of the right dimension. Moreover, since in the degree considered through a generic point of $\Delta_{(r, d-r), F}$ there is just one $r$ osculating line, the map $\tilde{p_{1}}$ is a desingularization of $\Delta_{(r, d-r), F}$. We will now recall how to compute the canonical bundle of $\tilde{\Delta}_{(r, d-r), F}$. As remarked in V2, the Picard group of $Z$ is generated by $H_{1}, H_{2}$ and $L$, the canonical class of $Z$ is $K_{Z}=-2 H_{1}-2 H_{2}+(-n+1) L$, and the class of $\mathcal{L}_{(r, d-r), F}$ is given by $r H_{1}+(d-r) H_{2}$. Therefore, by adjunction, we have

$K_{\tilde{\Delta}_{(r, d-r), F}}=K_{Z}+c_{1}\left(\mathcal{F}_{r, d-r}\right)=(r-2) H_{1}+(d-r-2) H_{2}+\left(\frac{d(d-1)}{2}-n+1\right) L$.

Consider now the bundles $\mathcal{N}_{Z}^{r, d-r}$ and $\mathcal{M}_{Z}^{d}$ on $Z$ respectively defined by the following two exact sequences:

$$
\begin{gathered}
0 \rightarrow \mathcal{N}_{Z}^{r, d-r} \rightarrow S^{d} \otimes \mathcal{O}_{Z} \rightarrow \mathcal{F}_{r, d-r} \rightarrow 0 \\
0 \rightarrow \mathcal{M}_{Z}^{d} \rightarrow S^{d} \otimes \mathcal{O}_{Z} \rightarrow \mathcal{E}_{d} \rightarrow 0 .
\end{gathered}
$$

By definition we have

$$
0 \rightarrow \mathcal{M}_{Z}^{d} \rightarrow \mathcal{N}_{Z}^{r, d-r} \rightarrow \mathcal{L}_{r, d-r} \rightarrow 0
$$

The needed positivity result is the following lemma. 
Lemma 4.1. If $r \geq 3, d-r \geq 2$ and

$$
\frac{d(d-1)}{2}-n \geq c-1
$$

then the bundle $\wedge^{c} \mathcal{M}_{Z \mid \tilde{\Delta}_{(r, d-r), F}}^{d} \otimes K_{\tilde{\Delta}_{(r, d-r), F}}\left(-H_{1}\right)$ is generated by its global sections.

Proof. Observe that $\mathcal{M}_{Z}^{d}=f^{*} M_{G}^{d}$. Hence, by Lemma 2.1, (ii), the bundle

$$
\begin{aligned}
& \bigwedge^{c} \mathcal{M}_{Z}^{d} \otimes \operatorname{det} \mathcal{F}_{r, d-r} \otimes K_{Z}\left(-H_{1}\right) \\
= & f^{*}\left(\bigwedge^{c} M_{G}^{d}\right) \otimes \mathcal{O}_{Z}\left((r-3) H_{1}+(d-r-2) H_{2}+\left(\frac{d(d-1)}{2}-n+1\right) L\right) \\
= & f^{*}\left(\bigwedge^{c} M_{G}^{d}(c)\right) \otimes \mathcal{O}_{Z}\left((r-3) H_{1}+(d-r-2) H_{2}+\left(\frac{d(d-1)}{2}-n-c+1\right) L\right)
\end{aligned}
$$

is globally generated under our numerical hypothesis, and the same holds for its restriction to $\tilde{\Delta}_{(r, d-r), F}$.

Now let $\Delta_{r, d-r} \subset \mathbf{P}^{n} \times S^{d}$ be the family of the $\Delta_{(r, d-r), F}$ 's, and $\tilde{\Delta}_{r, d-r} \subset$ $Z \times S^{d}$ the family of the desingularizations. Let $\mathcal{Y} \subset \tilde{\Delta}_{r, d-r}$ be a subscheme of relative dimension $k$, invariant under the action of $G L(n+1)$, and $\tilde{\mathcal{Y}} \rightarrow \mathcal{Y}$ a desingularization. Assume $h^{0}\left(\tilde{Y}_{F}, K_{\tilde{Y}_{F}}\left(-H_{1}\right)\right)=0$ and set $c=n-1-k$. Recall the isomorphisms

$$
\begin{aligned}
\bigwedge^{c} T \tilde{\Delta}_{r, d-r \mid \tilde{\Delta}_{(r, d-r), F}} \otimes K_{\tilde{\Delta}_{(r, d-r), F}} & \cong \Omega_{\tilde{\Delta}_{r, d-r}+\tilde{\Delta}_{(r, d-r), F}}^{N+k}, \\
\Omega_{\tilde{\mathcal{Y}}}^{N+k} \mid \tilde{Y}_{F} & \cong K_{\tilde{Y}_{F}},
\end{aligned}
$$

and consider the natural map

$$
\bigwedge^{c} T \tilde{\Delta}_{r, d-r \mid \tilde{\Delta}_{(r, d-r), F}} \otimes K_{\tilde{\Delta}_{(r, d-r),}} \underset{\bar{F}}{\cong} \Omega_{\tilde{\Delta}_{r, d}+k}^{N+k} \underset{\tilde{\Delta}_{(r, d-r), F}}{\rightarrow} \Omega_{\tilde{\mathcal{Y}}}^{N+k} \underset{\mid \tilde{Y}_{F}}{\cong} K_{\tilde{Y}_{F}} .
$$

If we twist (22) by $-H_{1}$, then, by assumption, the induced map in cohomology

$$
H^{0}\left(\bigwedge^{c} T \tilde{\Delta}_{r, d-r} \tilde{\Delta}_{(r, d-r), F} \otimes K_{\tilde{\Delta}_{(r, d-r), F}}\left(-H_{1}\right)\right) \rightarrow H^{0}\left(K_{\tilde{Y}_{F}}\left(-H_{1}\right)\right)
$$

is zero. Let $T_{\tilde{\Delta}_{r, d-r}}^{\text {vert }}$ be the sheaf defined by

$$
0 \rightarrow T_{\tilde{\Delta}_{r, d-r}}^{\text {vert }} \rightarrow T \tilde{\Delta}_{r, d-r} \rightarrow T Z \rightarrow 0 .
$$

Its restriction to $\tilde{\Delta}_{(r, d-r), F}$ coincides with $\mathcal{N}_{Z}^{r, d-r} \tilde{\Delta}_{(r, d-r), F}$. Therefore, by (18) and lemma 4.1, we have constructed a subbundle

$$
\begin{aligned}
\bigwedge^{c} \mathcal{M}_{Z \mid \tilde{\Delta}_{(r, d-r), F}}^{d} \otimes K_{\tilde{\Delta}_{(r, d-r), F}}\left(-H_{1}\right) \\
\hookrightarrow \bigwedge^{c} T^{v e r t} \tilde{\Delta}_{r, d-r} \tilde{\Delta}_{(r, d-r), F} \otimes K_{\tilde{\Delta}_{(r, d-r), F}}\left(-H_{1}\right),
\end{aligned}
$$

which is generated by its global sections, under the numerical hypothesis of Lemma 4.1 .

We conclude Case 1 with the following fact.

Proposition 4.2. Let $F$ be a general polynomial of degree $d$ satisfying (19), with $c=n-1-k$. Suppose $r \geq 2$ and $d-r \geq 2$. Let $Y_{F} \subset \tilde{\Delta}_{(r, d-r), F}$ be a subvariety of dimension $k$, and $j: \tilde{Y}_{F} \rightarrow Y_{F}$ a desingularization such that $h^{0}\left(\tilde{Y}_{F}, K_{\tilde{Y}_{F}}\left(-j^{*} H_{1}\right)\right)=$ 0 . Then $Y_{F}$ is contained in the locus of lines of $X_{F}$. 
Proof. When no confusion is possible, we will omit the index $(r, d-r)$ in what follows, and simply set $\Delta=\Delta_{r, d-r}$. Suppose first that $r \geq 3$. Let $W \subset T_{\tilde{\Delta},(z, F)}$ be a codimension $c$ subspace contained in the base locus of $H^{0}\left(\bigwedge^{c} T \tilde{\Delta}_{\mid \tilde{\Delta}_{F}} \otimes K_{\tilde{\Delta}_{F}}\left(-H_{1}\right)\right)$, considered as the space of sections of a line bundle on the Grassmannian of codimension $c$ subspaces of $T \tilde{\Delta}_{\mid \tilde{\Delta}_{F}}$. Then we must have

$$
W^{\text {vert }}:=\left.\left.W \cap \mathcal{N}_{Z}^{r, d-r}\right|_{z} \subset \mathcal{M}_{Z}^{d}\right|_{z} .
$$

Indeed, if this were not the case, we would have $\operatorname{codim}_{\left.\mathcal{M}_{Z}^{d}\right|_{z}} \bar{W}=c$, where $\bar{W}:=$ $\left.W \cap \mathcal{M}_{Z}^{d}\right|_{z}$. Then consider the following commutative diagram:

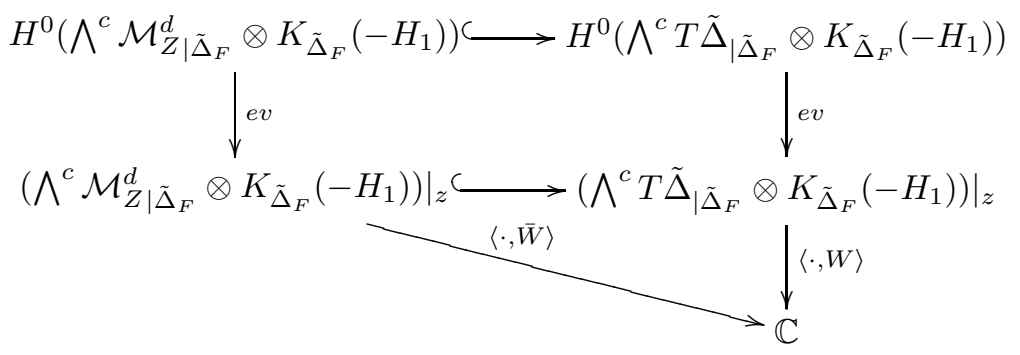

(ev is the evaluation of the sections at the point $z$, and $\langle\cdot, W\rangle$ is the contraction defined by the subspace $W)$. Since $W$ belongs to the base locus of $H^{0}\left(\bigwedge^{c} T \tilde{\Delta}_{\mid \tilde{\Delta}_{F}} \otimes\right.$ $\left.K_{\tilde{\Delta}_{F}}\left(-H_{1}\right)\right)$, then the composite map $\langle\cdot, W\rangle \circ \mathrm{ev}$ is zero, and so would be $\langle\cdot, \bar{W}\rangle \circ \mathrm{ev}$. But this is absurd, because, by Lemma 4.1 the bundle $\bigwedge^{c} \mathcal{M}_{Z \mid \tilde{\Delta}_{F}}^{d} \otimes K_{\tilde{\Delta}_{F}}\left(-H_{1}\right)$ is generated by its global sections.

So let $\mathcal{Y} \subset \tilde{\Delta}$ be a subvariety which is stable under the action of $G L(n+1)$ and of relative codimension $c$. Assume moreover that the restriction map (23) is zero. By (24), $T_{\mathcal{Y},(z, F)}^{v e r t}$ is contained in

$$
\mathcal{M}_{Z \mid z}^{d}=\left\{G \in S^{d}: G_{\mid \ell_{z}}=0\right\} .
$$

On the other hand, by Remark 2.0.1, (ii), $T_{\mathcal{Y},(z, F)}^{v e r t}$ contains $F$ itself. So by (26) we have that $F_{\mid \ell_{z}}=0$ for every point $z \in Y_{F}$, i.e. $Y_{F}$ is contained in the subvariety covered by the lines contained in $X_{F}$.

If $r=2$, we can consider the natural isomorphism

$$
\tilde{\Delta}_{(r, d-r), F} \stackrel{\sim}{\longrightarrow} \tilde{\Delta}_{(d-r, r), F}
$$

sending a point $z \in \tilde{\Delta}_{(r, d-r), F}$ with $b(z)=\left(x, x^{\prime}\right)$ to a point $w \in \tilde{\Delta}_{(d-r, r), F}$ with $b(w)=\left(x^{\prime}, x\right)$, where $x$ and $x^{\prime}$ are points on $X_{F}$ linked by the condition

$$
\exists \text { a line } \ell \text { s.t. } \ell \cap X_{F}=r \cdot x+(d-r) \cdot x^{\prime} .
$$

Since $r=2$ implies $d-r \geq 3$, from what we have done before it follows that the conclusion is true for $\tilde{\Delta}_{(d-r, r), F}$, and so, by (27), the same holds for $\tilde{\Delta}_{(r, d-r), F}$.

Case 2: $r=1$ or $d-r=1$.

Suppose for instance $d-r=1$. Let $\Gamma \subset \mathbf{P}^{n} \times \operatorname{Gr}(1, n)$ be the incidence variety, and $p$ and $q$ the projections on the two factors. Let $\pi: \mathbf{P} \rightarrow \Gamma$ be the pull-back of the universal $\mathbf{P}^{1}$-bundle over $\operatorname{Gr}(1, n)$ and $\tau$ the natural section of $\pi$. Consider the bundle $\mathcal{E}_{d}:=\pi_{*} \mathcal{O}_{\mathbf{P}}(d)$ over $\Gamma$, and its rank 2 subbbundle $\mathcal{K} \subset \mathcal{E}_{d}$ such that its fiber 
at a point $(x, \ell)$ is given by the polynomials $P \in H^{0}\left(\mathcal{O}_{\ell}(d)\right)$ vanishing to order at least $(d-1)$ at $x$. Consider the line bundle $\mathcal{L}_{1}$ defined by

$$
0 \rightarrow \mathcal{L}_{1} \rightarrow \mathcal{E}_{1} \rightarrow \tau^{*} \mathcal{O}_{\mathbf{P}}(1)=: H \rightarrow 0 .
$$

Then $\mathcal{K} \cong \mathcal{L}_{1}^{d-1} \otimes \mathcal{E}_{1}$. Let $\mathcal{F}_{d}$ be the quotient $\mathcal{E}_{d} / \mathcal{K}$. As in Case 1 , to any $F \in S^{d}$ we can associate a global section $\sigma_{F}$ of $\mathcal{F}_{d}$. By definition $p\left(V\left(\sigma_{F}\right)\right)=\Delta_{(d-1,1), F}$. As before, since the bundle $\mathcal{F}_{d}$ is generated by the sections $\sigma_{F}$, we have that $V\left(\sigma_{F}\right)$ is smooth of the right dimension, for a general $F$, and it is easy to verify that $p: V\left(\sigma_{F}\right) \rightarrow \Delta_{(d-1,1), F}$ is a desingularization. Then we define $\tilde{\Delta}_{(d-1,1), F}:=$ $V\left(\sigma_{F}\right)$. Hence, using the adjunction formula, we can compute the canonical bundle of $\tilde{\Delta}_{(d-1,1), F}$ as the restriction to $\tilde{\Delta}_{(d-1,1), F}$ of the following line bundle:

$$
K_{G r(1, n)}+c_{1}\left(\mathcal{F}_{d}\right)=(2 d-4) H+\left(\frac{d(d+1)}{2}-n-1-2(d-1)\right) L .
$$

Consider the bundles $\mathcal{N}_{\Gamma}^{d}$ and $\mathcal{M}_{\Gamma}^{d}$ on $\Gamma$ respectively defined by the two following exact sequences:

$$
\begin{aligned}
& 0 \rightarrow \mathcal{N}_{\Gamma}^{d} \rightarrow S^{d} \otimes \mathcal{O}_{\Gamma} \rightarrow \mathcal{F}_{d} \rightarrow 0, \\
& 0 \rightarrow \mathcal{M}_{\Gamma}^{d} \rightarrow S^{d} \otimes \mathcal{O}_{\Gamma} \rightarrow \mathcal{E}_{d} \rightarrow 0 .
\end{aligned}
$$

From the definitions it follows that we have

$$
0 \rightarrow \mathcal{M}_{\Gamma}^{d} \rightarrow \mathcal{N}_{\Gamma}^{d} \rightarrow \mathcal{K} \rightarrow 0 .
$$

The positivity result we will need this time is the following:

Lemma 4.3. If

$$
\frac{d(d+1)}{2}-n-1-2(d-1) \geq c-1,
$$

then the bundle $\bigwedge^{c} \mathcal{M}_{\Gamma \mid \tilde{\Delta}_{(d-1,1), F}}^{d} \otimes K_{\tilde{\Delta}_{(d-1,1), F}}(-H)$ is generated by its global sections.

Proof. Use (28) and Lemma 2.1 (ii).

Now let $\Delta_{d-1,1} \subset \mathbf{P}^{n} \times S^{d}$ be the family of the $\Delta_{(d-1,1), F}$ 's, and $\tilde{\Delta}_{d-1,1} \subset$ $\Gamma \times S^{d}$ the family of the desingularizations. Let $\mathcal{Y} \subset \tilde{\Delta}_{r, d-r}$ be a subscheme of relative dimension $k$, invariant under the action of $G L(n+1)$, and $\tilde{\mathcal{Y}} \rightarrow \mathcal{Y}$ a desingularization. Consider the sheaf $T_{\tilde{\Delta}_{d-1,1}}^{\text {vert }}$ defined by

$$
0 \rightarrow T_{\tilde{\Delta}_{d-1,1}}^{v e r t} \rightarrow T_{\tilde{\Delta}_{r, d-r}} \rightarrow T_{\Gamma} \rightarrow 0
$$

and observe that its restriction to $\tilde{\Delta}_{(d-1,1), F}$ coincides with $\mathcal{N}_{\Gamma \mid \tilde{\Delta}_{(d-1,1), F}}^{d}$. If we assume $h^{0}\left(\tilde{Y}_{F}, K_{\tilde{Y}_{F}}(-H)\right)=0$ and set $c=n-1-k$, then the natural adjunction map

$$
H^{0}\left(\bigwedge^{c} \mathcal{M}_{\Gamma_{\mid \tilde{\Delta}_{(d-1,1), F}}^{d}} \otimes K_{\tilde{\Delta}_{(d-1,1), F}}(-H)\right) \rightarrow H^{0}\left(\tilde{Y}_{F}, K_{\tilde{Y}_{F}}(-H)\right)
$$

which we can construct thanks to (31), is obviously zero.

The last step will be the proof of the following:

Proposition 4.4. Let $F$ be a general polynomial of degree d satisfying (32). Let $Y_{F} \subset \tilde{\Delta}_{(d-1,1), F}$ be a subvariety of dimension $k$, and $j: \tilde{Y}_{F} \rightarrow Y_{F}$ a desingularization such that (33) vanishes. Then $Y_{F}$ is contained in the locus of lines of $X_{F}$. 
Proof. Recall that $F \in T^{\text {vert }} \mathcal{Y}_{\mid(x, \ell, F)}$. We claim that

$$
F \in \mathcal{M}_{\Gamma \mid(x, \ell)}^{d} .
$$

Indeed if $F \in \mathcal{K}_{\mid(x, \ell)}$, then we have the surjection

$$
T^{\text {vert }} \mathcal{Y}_{\mid(x, \ell, F)} \rightarrow \mathcal{K}_{\mid(x, \ell)} .
$$

(This follows from the fact that if $F \in \mathcal{K}_{\mid(x, \ell)}$ then

$$
\left\langle S_{x}^{1} \cdot J_{F}^{d-1}, F\right\rangle \rightarrow \mathcal{K}_{\mid(x, \ell)},
$$

plus Remark 2.0.1, (ii)). Then, by (34),

$$
\operatorname{codim}_{\mathcal{M}_{\Gamma \mid}^{d}(x, \ell)} T^{\text {vert }} \mathcal{Y}_{\mid(x, \ell, F)}=\operatorname{codim}_{X_{F}} Y_{F}=c .
$$

As in Proposition 4.2, we can now use the commutative diagram

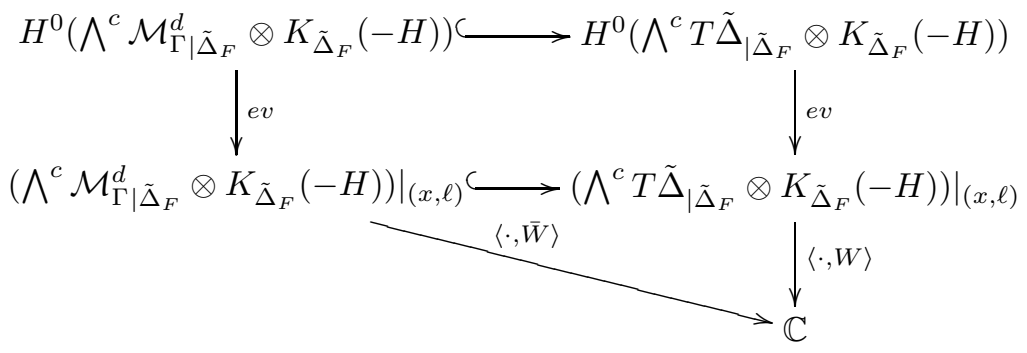

and deduce from it, together with Lemma 4.3 and Remark 2.0.1, (ii), that what we claim holds, i.e.

$$
F_{\mid \ell}=0 \text {. }
$$

The numerical condition (32), which for $c=n-1-k$ becomes

$$
\frac{d(d-3)}{2} \geq 2 n-k-3,
$$

implies (19). Thus, combining Propositions 4.2 and 4.4 the proof of our main theorem is completed.

\section{ACKNOWLEDGEMENTS}

I would like to thank M. Zaidenberg and L. Manivel, who invited me, respectively in February 2001 and March 2002, to the Institut Fourier in Grenoble, where the preliminary version of this work was started and finished. My sincerest thanks to H. Clemens, who sent me the manuscript $\mathrm{C} 2$ ] and kindly encouraged me when I communicated to him the idea of adapting some arguments contained in $\mathrm{C} 2$ to strengthten my work. Finally, it is a pleasure to acknowledge the great influence of C. Voisin's articles [V1] and [V2] on my work.

\section{REFERENCES}

[C1] H. Clemens, Curves in generic hypersurfaces, Ann. Sci. École Norm. Sup. 19 (1986), 629636; erratum, ibid. 20 (1987), 281. MR 88c:14037, MR 88i:14017

[C2] H. Clemens, Limiting the genus of subvarieties of generic hypersurfaces, Comm. in Algebra, Special issue in honour of S. Kleiman, to appear.

[CL] L. Chiantini, A. F. Lopez, Focal loci of families and the genus of curves on surfaces, Proc. Amer. Math. Soc. 127 (1999), no. 12, 3451-3459. MR 2000e:14057

[CLR] L. Chiantini, A. F. Lopez and Z. Ran, Subvarieties of generic hypersurfaces in any variety, Math. Proc. Cambr. Phil. Soc., 130 (2001), no. 2, 259-268. MR 2002d:14066 
[CR] H. Clemens, Z. Ran, On the geometric genus of subvarieties of generic hypersurfaces, preprint math.AG/0204256.

[E1] L. Ein, Subvarieties of generic complete intersections, Invent. Math. 94 (1988), 163-169. MR 89i:14002

[E2] L. Ein, Subvarieties of generic complete intersections II, Math. Ann. 289 (1991), 465-471. MR 92h:14002

[L] S. Lang, Hyperbolic and Diophantine analysis, Bull. Amer. Math. Soc. (N.S.) 14 (1986), no. 2, 159-205. MR 87h:32051

[P] G. Pacienza, Rational curves on general projective hypersurfaces, J. of Alg. Geometry 12 (2003), 245-267. MR 2003k:14065

[V1] C. Voisin, On a conjecture of Clemens on rational curves on hypersurfaces, J. of Diff. Geometry 44 (1996), 200-214. MR 97j:14047

[V2] C. Voisin, A correction "On a conjecture of Clemens on rational curves on hypersurfaces", J. of Diff. Geometry 49 (1998), 601-611. MR 99m:14078

[X1] G. Xu, Subvarieties of general hypersurfaces in projective space, J. of Differential Geom. 39 (1994), no. 1, 139-172. MR 95d:14043

[X2] G. Xu, Divisors on generic complete intersection in projective space, Trans. A.M.S. 348 (1996), 2725-2736. MR 96i:14041

Institut de Mathématiques de Jussieu, Université Pierre et Marie Curie, 4, Place Jussieu, F-75252 Paris Cedex 05, France

E-mail address: pacienza@math.jussieu.fr

Current address: IRMA - Université Louis Pasteur et CNRS, 7, Rue R. Descartes, 67084 Strasbourg Cedex, France

E-mail address: pacienza@math.u-strasbg.fr 